Preprint Numbers: ANL-PHY-7663-TH-93

UNITUE-THEP-13/1993

\title{
Pion Loop Contribution to the Electromagnetic Pion Charge Radius
}

\author{
Reinhard Alkofer*, Axel Bender* and Craig D. Roberts ${ }^{\dagger}$ \\ * Institut für Theoretische Physik, Universität Tübingen, \\ Auf der Morgenstelle 14, D-72076 Tübingen, FRG \\ ${ }^{\dagger}$ Physics Division, Argonne National Laboratory, \\ Argonne, Illinois 60439-4843, USA
}

\begin{abstract}
A phenomenological Dyson-Schwinger equation approach to QCD, formalised in terms of a QCD based model field theory, is used to calculate the electromagnetic charge radius of the pion. The contributions from the quark core and pion loop, as defined in this approach, are identified and compared. It is shown explicitly that the divergence of the charge radius in the chiral limit is due to the pion loop and that, at the physical value of the pion mass, this loop contributes less than $15 \%$ to $\left\langle r_{\pi}^{2}\right\rangle$; i.e. the quark core is the dominant determining characteristic for the pion. This suggests that quark based models which fail to reproduce the $\ln m_{\pi}$ divergence of $\left\langle r_{\pi}^{2}\right\rangle$ may nevertheless incorporate the dominant characteristic of the pion: its quark core.
\end{abstract}

Pacs Numbers: 14.40.Aq, 13.40.Fn, 11.30.Rd, 12.40.Aa, 12.38.Lg

Typeset using REVTEX 


\section{INTRODUCTION}

Chiral symmetry and its dynamical breakdown is crucial to the nature of the strong interaction spectrum and the study of this in Quantum Chromodynamics (QCD), and models thereof, is of great current interest. The most striking signals of Dynamical Chiral Symmetry Breaking (DCSB) are found in the kinematic and dynamical properties of the pion and one tool that has been used extensively to study these properties is Chiral Perturbation Theory (ChPT) 1ㅐ. Chiral Perturbation Theory can be understood as the study of the necessary consequences of the chiral Ward Identities via the construction of an effective action, using field variables with pionic quantum numbers, in such a way as to ensure that these identities are realised. It should be noted that in this approach the pionic field has no physical significance - it is merely an auxiliary field - and should not be identified with the physical pion [5].

At first nonleading order in ChPT, $\mathrm{O}\left(\mathrm{E}^{4}\right)$, the effective action is only completely determined once the effect of one-pion loops, generated by the $\mathrm{O}\left(\mathrm{E}^{2}\right)$ part of the action, is included. The regularisation of the divergence of each of these loops introduces ten arbitrary parameters at this level. The theory is not renormalisable but the approach is nevertheless useful because higher order loops do not contribute to the $\mathrm{O}\left(\mathrm{E}^{4}\right)$ action [6]; i.e., the action at $\mathrm{O}\left(\mathrm{E}^{4}\right)$ is completely specified once the effect of the $\mathrm{O}\left(\mathrm{E}^{2}\right)$ loops is taken into account and the 10 parameters fixed by comparison with experimental data.

The pion (or, more generally, pseudoscalar) loops in ChPT are characteristic of the approach and, indeed, are sometimes regarded as being the dominant feature. The expression for every physical observable receives a contribution from such loops which depends on the mass of the particle in the loop and which diverges in the chiral limit. For example, in the case of the electromagnetic pion charge radius the loop introduces a logarithmic dependence and hence a logarithmic divergence in the chiral limit. Herein we are interested in estimating the importance of these loop contributions, evaluated at real pseudoscalar masses, relative to what one might call the "quark core" contribution and our exemplary case will be the electromagnetic charge radius of the pion.

The pseudoscalar mesons are the simplest bound states to study within the coupled Dyson-Schwinger-Bethe-Salpeter equation approach to QCD, recent reviews of which can be found in Refs. [7 9]. In vector exchange theories with DCSB, such as QCD, the Goldstoneboson character of the pseudoscalar excitations can be understood through the existence of an identity between the fermion Dyson-Schwinger equation (DSE) and the pseudoscalar Bethe-Salpeter equation (BSE). If, in the limit $P^{2} \rightarrow 0$, this BSE reduces to the DSE then DCSB necessarily entails the existence of massless pseudoscalar excitations. This result was established for the rainbow-DSE-ladder-BSE pair in Ref. [10]. (The rainbow approximation in the DSE is equivalent to the ladder approximation in the BSE.) In this approach, the "quark core" of a meson is described by the Bethe-Salpeter amplitude obtained as a solution of the pesudoscalar BSE; the kernel of which, in practice, is often constructed as a generalised ladder kernel: generalised in the sense that the quark propagators are obtained as solutions of the rainbow DSE with a gluon propagator whose large spacelike- $q^{2}$ behaviour is that given by the renormalisation group in QCD and whose small spacelike- $q^{2}$ behaviour is modelled so as to ensure confinement [11 [14].

The coupled rainbow-DSE-ladder-BSE approach to modelling QCD is equivalent to the 
Global Colour-symmetry Model (GCM), first described in Ref. [15] and extended to the pseudoscalar sector in Refs. [16,17]. The extension to other mesons and baryons is reviewed in Ref. [18]. As discussed in Ref. [17], this approach provides a very good description of kinematic and dynamical properties of pions. For example, using the quark core alone, it describes $\pi-\pi$ partial wave amplitudes very well up to $600 \mathrm{MeV}$; i.e., from threshold up to the first resonance. As indicated above, the parameters in the GCM describe the quark-quark interaction at small spacelike- $q^{2}$.

The model represents QCD as an effective two-point theory, coloured quark currents interact via dressed gluon exchange, and has the "disadvantage" that there is some model dependence in the results: for example, it predicts values for the parameters that appear in the effective lagrangian of ChPT [17,19]. However, since these parameters have been fitted to low energy phenomena in QCD, this provides a means of checking the model assumptions in the GCM. Thus the model dependence is also an advantage: the assumptions on which the model is founded can be tested and this enables the development of an understanding of hadronic phenomena in terms of quark and gluon interactions.

Herein, in the context of the GCM, we calculate the electromagnetic charge radius of the pion taking into account both the "quark core" and the pion-loop rescattering correction. The "quark core" contribution, obtained in a generalised impulse approximation [17,20, 21], is regular in the chiral limit whereas, as noted above, the pion-loop contribution is not. Our aim is to estimate which is the dominant piece at the physical pion mass and hence to infer whether the electromagnetic structure of the pion is dominated by the "quark core" or a "pseudoscalar meson-cloud".

In Sec. II we describe the calculation of $\left(r_{\pi}^{2}\right)^{\text {GIA }}$, the generalised impulse approximation "quark core" contribution to the charge radius. In Sec. III we discuss the calculation of the pion loop contribution to the charge radius. We put these pieces together to obtain the net $\pi \pi \gamma$ vertex and obtain an expression for the net charge form factor in Sec. IV. Our results are presented and discussed in Sec. V. We summarise and conclude in Sec. VI.

\section{GENERALISED IMPULSE APPROXIMATION}

In Euclidean space, with metric $\delta_{\mu \nu}=\operatorname{diag}(1,1,1,1), \gamma_{\mu}$ hermitian and $m_{u}=m_{d}$, we define the generalised impulse approximation contribution to the connected $\pi \pi \gamma$ vertex to be: 17,20,21]

$$
\begin{aligned}
& \Lambda_{\mu}^{\mathrm{GIA}}(p, q)= \\
& \frac{N_{c}}{f_{\pi}^{2}} \int \frac{d^{4} k}{(2 \pi)^{4}} \operatorname{tr}_{D}\left[\bar{\Gamma}_{\pi}\left(k_{0+} ; p\right) S\left(k_{++}\right) i \Gamma_{\mu}\left(k_{++}, k_{--}\right) S\left(k_{--}\right) \Gamma_{\pi}\left(k_{-0} ; q\right) S\left(k_{-+}\right)\right] .
\end{aligned}
$$

In this expression the trace over colour and flavour indices has been carried out leaving only the trace over Dirac indices and the definition

$$
k_{\alpha \beta}=k+\frac{\alpha}{2} p+\frac{\beta}{2} q
$$

has been used. The dressed quark-photon vertex is denoted by $\Gamma_{\mu}\left(p_{1}, p_{2}\right)$, the pion BetheSalpeter amplitude by $\Gamma_{\pi}(p ; P)$ and the dressed quark propagator by $S(p)$. This vertex is illustrated in Fig. [ 1 and is what we mean by the "quark core" contribution to the electromagnetic properties of the pion. 


\section{A. Quark propagator}

We describe equation (11) as "generalised impulse approximation" for a number of reasons. First, the internal quark lines are described by dressed quark propagators that are obtained by solving the quark Dyson-Schwinger equation (DSE):

$$
S^{-1}(p)=i \gamma \cdot p+m+\frac{4}{3} g^{2} \int \frac{d^{4} k}{(2 \pi)^{4}} \gamma_{\mu} S(k) \Gamma_{\nu}^{g}(k, p) D_{\mu \nu}\left((p-k)^{2}\right) .
$$

In this equation $D_{\mu \nu}(k)$ is the dressed gluon propagator and $\Gamma_{\nu}^{g}\left(p_{1}, p_{2}\right)$ is the dressed quarkgluon vertex; each of these quantities satisfies its own DSE. The general solution of this equation has the form

$$
S(p)=-i \gamma \cdot p \sigma_{V}\left(p^{2}\right)+\sigma_{S}\left(p^{2}\right)
$$

A commonly used equivalent representation is to specify the inverse in terms of two scalar functions:

$$
S^{-1}(p)=i \gamma \cdot p A\left(p^{2}\right)+m+B\left(p^{2}\right)
$$

where $m$ is the current quark mass. Both forms will be used herein. The commonly used "rainbow approximation" DSE is obtained from Eq. (3) by writing

$$
\Gamma_{\nu}^{g}(k, p)=\gamma_{\nu}
$$

Equation (3) has been studied extensively using many model forms for the dressed gluon propagator and quark-gluon vertex and some of this work is summarised in Refs. [7 9]. A good deal has been learnt about the analytic properties of the quark propagator in the complex plane [22,23] and this is important in the calculation of many observables. In connection with the electromagnetic charge radius of the pion, however, it is sufficient to have a representation (or interpolation) of the quark propagator that is valid on the real spacelike axis. One such simple form, which is a modification of that used in Ref. 24] and a simplification of the form used in Ref. [17], is

$$
\begin{aligned}
& A\left(p^{2}\right)=1, \\
& B\left(p^{2}\right)=\frac{\Lambda_{1}^{3}}{p^{2}+\Lambda_{2}^{2}},
\end{aligned}
$$

where reasonable values of the parameters are $\Lambda_{1} \sim 0.5 \mathrm{GeV} \sim \Lambda_{2}$. We remark that the fact that $B \neq 0$, even when the quark bare mass is zero, is a manifestation of DCSB and, in general, for the small quark bare masses that are appropriate for the pion sector, $A\left(p^{2}\right)$ and $B\left(p^{2}\right)$ are insensitive to $m$; a result that can be seen clearly in the model of Ref. [23]. This means that in our parametrisation of the DSE results $\Lambda_{1}$ and $\Lambda_{2}$ are also insensitive to $\mathrm{m}$. We emphasise that these forms are only suitable for interpolation on the real spacelike axis. We will discuss this Ansatz in more detail below [see Sec. IIC]. 


\section{B. Pion Bethe-Salpeter amplitude}

The generalised impulse approximation, in addition to using dressed quark propagators, uses a pion Bethe-Salpeter amplitude that is obtained as the solution of the homogeneous Bethe-Salpeter equation (BSE):

$$
\Gamma_{\pi}^{r s}(p ; P)=\int \frac{d^{4} k}{(2 \pi)^{4}} K^{r s ; t u}(k, p ; P)\left(S\left(k-\frac{1}{2} P\right) \Gamma_{\pi}(k ; P) S\left(k+\frac{1}{2} P\right)\right)^{t u}
$$

where $P$ is the centre-of-mass momentum of the bound state, $p$ is the relative momentum between the quarks in the bound state, and the superscripts, which run from 1-4, are associated with the Dirac structure of the amplitude. The kernel, $K^{r s ; t u}(p, k ; P)$, has no flavour structure in the isospin symmetric limit $m_{u}=m_{d}$ and since $\Gamma_{\pi}(p ; P) \propto I_{C}$ and $S(p) \propto I_{C}$ then $K^{r s ; t u}(p, k ; P) \propto I_{C}$, where $I_{C}$ is the identity in colour space. The ladder approximation BSE is obtained from Eq. (9) by writing

$$
K^{r s ; t u}(k, p ; P)=\frac{4}{3} g^{2} D_{\mu \nu}(p-k)\left(\gamma_{\mu}\right)^{r t}\left(\gamma_{\mu}\right)^{u s}
$$

Here $g^{2} D_{\mu \nu}$ is a model dressed gluon propagator which, in Landau gauge, has the form:

$$
\frac{g^{2}}{4 \pi} D_{\mu \nu}(k)=\left(\delta_{\mu \nu}-\frac{k_{\mu} k_{\nu}}{k^{2}}\right) D\left(k^{2}\right) .
$$

The behaviour of $D\left(k^{2}\right)$ at large spacelike- $k^{2}$ is determined from the QCD renormalisation group:

$$
D\left(k^{2}\right) \stackrel{k^{2} \rightarrow \infty}{\approx} \frac{1}{k^{2}} \frac{\lambda \pi}{\ln \frac{k^{2}}{\Lambda_{\mathrm{QCD}}^{2}}}
$$

with $\lambda=12 /\left[33-2 N_{f}\right]$ and where $\Lambda_{\mathrm{QCD}} \approx 0.2 \mathrm{GeV}$ is the renormalisation point invariant QCD mass scale. The behaviour of $D\left(k^{2}\right)$ at small and intermediate spacelike- $k^{2}$ is unknown and in the GCM [or coupled DSE-BSE approach] a few parameters are used to specify a model form; i.e., the behaviour of the quark-quark interaction in the infrared is parametrised. The results one obtains for physical quantities in this approach are completely determined by the values of these parameters, as emphasised in Ref. [17]. The constraints and commonly used parametrisations are discussed in detail in Refs. [7 9] and one efficacious form has

$$
D\left(q^{2}\right)=C \Lambda_{\mathrm{QCD}}^{2} \delta^{4}(q)+\frac{\lambda \pi}{q^{2} \ln \left(\tau+\frac{q^{2}}{\Lambda_{\mathrm{QCD}}^{2}}\right)}
$$

with $C=(3 \pi)^{3}$ and $\tau=3[11]$.

The most general form of the pseudoscalar amplitude, allowed by Lorentz covariance, which is odd under parity transformations is [25]

$$
\Gamma_{\pi}(p ; P)=\gamma_{5}\{E(p, P)-i(\gamma \cdot p)(p \cdot P) F(p, P)-i(\gamma \cdot P) G(p, P)-[\gamma \cdot p, \gamma \cdot P] H(p, P)\}
$$


and since $\pi^{0}$ is even under charge-conjugation then $E, F, G$ and $H$ are even functions of $(p \cdot P)$ in this case.

The coupled rainbow-DSE-ladder-BSE system, Eq. (3) with (6) plus Eq. (9) with (10), which is equivalent to the GCM effective action approach to QCD, has been studied extensively and provides an excellent description of light-light, heavy-light and heavy-heavy meson systems [11 14. These studies show that it is a good approximation to write

$$
\Gamma_{\pi}(p ; P)=\gamma_{5} E(p, P) .
$$

In the chiral limit; i.e., when the current quark mass, $m$, is zero, the pseudoscalar ladder-BSE and quark rainbow-DSE are identical [10] and one has a massless excitation in the pseudoscalar channel with

$$
E\left(p, P^{2}=0\right) \propto B\left(p^{2}\right)
$$

if the quark DSE allows a dynamically generated mass function: $B\left(p^{2}\right) \neq 0$; i.e, if there is DCSB. This is the manner in which Goldstone's theorem is realised in the GCM and coupled DSE-BSE approach. In this case the parametrisation of Eq. (8) is also a good representation of the pion Bethe-Salpeter amplitude.

The normalisation of the amplitude is fixed, as usual, by requiring that, for $P^{2}=-m_{\pi}^{2}$ :

$$
\begin{aligned}
& 2 f_{\pi}^{2} P_{\mu}=\int \frac{d^{4} k}{(2 \pi)^{4}} \frac{d^{4} q}{(2 \pi)^{4}} \operatorname{tr}_{D}\left[\bar{\Gamma}_{\pi}(k ; P) \frac{\partial K(k, q ; P)}{\partial P_{\mu}} \Gamma_{\pi}(q ;-P)\right]+ \\
& N_{c} \int \frac{d^{4} k}{(2 \pi)^{4}} \operatorname{tr}_{D}\left[\bar{\Gamma}_{\pi}(k ; P) S\left(k_{0-}\right) \Gamma_{\pi}(k ;-P) \frac{\partial S\left(k_{0+}\right)}{\partial P_{\mu}}+\bar{\Gamma}_{\pi}(k ; P) \frac{\partial S\left(k_{0-}\right)}{\partial P_{\mu}} \Gamma_{\pi}(k ;-P) S\left(k_{0+}\right)\right] .
\end{aligned}
$$

If the Bethe-Salpeter interaction kernel, $K(k, p ; P)$, is independent of the centre-of-mass momentum, $P$, which is the case in ladder approximation, for example, then this reduces to

$$
\begin{aligned}
& 2 f_{\pi}^{2} P_{\mu}= \\
& N_{c} \int \frac{d^{4} k}{(2 \pi)^{4}} \operatorname{tr}_{D}\left[\bar{\Gamma}_{\pi}(k ; P) S\left(k_{0-}\right) \Gamma_{\pi}(k ;-P) \frac{\partial S\left(k_{0+}\right)}{\partial P_{\mu}}+\bar{\Gamma}_{\pi}(k ; P) \frac{\partial S\left(k_{0-}\right)}{\partial P_{\mu}} \Gamma_{\pi}(k ;-P) S\left(k_{0+}\right)\right] .
\end{aligned}
$$

\section{Quark-photon vertex}

The generalised impulse approximation contribution also involves the dressed quarkphoton vertex $\Gamma_{\mu}\left(p_{1}, p_{2}\right)$ which satisfies its own integral equation. Solving this equation is a difficult problem which has only recently begun to be addressed [26]. However, it is clear that if the quark propagator has momentum dependent dressing then $\Gamma_{\mu}\left(p_{1}, p_{2}\right)$ cannot be the bare vertex since the Ward Identity

$$
\left(p_{1}-p_{2}\right)_{\mu} i \Gamma_{\mu}\left(p_{1}, p_{2}\right)=S^{-1}\left(p_{1}\right)-S^{-1}\left(p_{2}\right)
$$

would not then be satisfied and hence the electromagnetic current of the pion would not be conserved. 
It is possible to learn much about this vertex without actually solving its integral equation. Indeed, it is argued in Refs. [27 30] that a "physically reasonable" Ansatz for this vertex must take the form:

$$
\Gamma_{\mu}(p, k)=\Gamma_{\mu}^{\mathrm{BC}}(p, k)+\Gamma_{\mu}^{\mathrm{T}}(p, k)
$$

where [31]

$$
\begin{aligned}
\Gamma_{\mu}^{\mathrm{BC}}(p, k) & =\frac{\left[A\left(p^{2}\right)+A\left(k^{2}\right)\right]}{2} \gamma_{\mu} \\
& +\frac{(p+k)_{\mu}}{p^{2}-k^{2}}\left\{\left[A\left(p^{2}\right)-A\left(k^{2}\right)\right] \frac{[\gamma \cdot p+\gamma \cdot k]}{2}-i\left[B\left(p^{2}\right)-B\left(k^{2}\right)\right]\right\}
\end{aligned}
$$

and

$$
(p-k)_{\mu} \Gamma_{\mu}^{\mathrm{T}}(p, k)=0 \text { with } \Gamma_{\mu}^{\mathrm{T}}(p, p)=0
$$

In the bare quark propagator limit, $A=1$ and $B=$ constant, $\Gamma_{\mu}^{\mathrm{T}}=0$. This Ansatz is "physically reasonable" because it satisfies the criteria a)-d) discussed in Ref. [30]: a) it satisfies the Ward-Takahashi identity; b) it is free of kinematic singularities; c) it reduces to the bare vertex in the free field limit; d) it has the same transformation properties as the bare vertex under charge conjugation and Lorentz transformations. The $\Gamma_{\mu}^{\mathrm{BC}}(p, k)$ piece is completely determined by the quark propagator but the remaining piece, $\Gamma_{\mu}^{\mathrm{T}}(k, p)$, is undetermined.

It is in this additional transverse piece that photon-vector-meson mixing contributions will appear; i.e., vector meson dominance contributions are confined to $\Gamma_{\mu}^{\mathrm{T}}(p, k)$. This is especially clear in the Nambu-Jona-Lasinio (NJL) model where $\Gamma_{\mu}^{\mathrm{BC}}(p, k)=\gamma_{\mu}$ [32,33.

We will define our generalised impulse approximation as Eq. (11) with

$$
\Gamma_{\mu}^{\mathrm{T}}(p, k) \equiv 0
$$

This allows for a very good description of the electromagnetic form factor in the spacelike region; i.e., away from resonance contributions [21]. We note that criterion c) is important because, in association with a solution of the DSE for the quark propagator which exhibits the correct perturbative QCD leading-log behaviour of $\mathrm{S}(\mathrm{p})$, which is guaranteed when the gluon propagator in Eq. (3) has the ultraviolet form given in Eq. (12), it ensures that the vertex Ansatz will yield the same large- $Q^{2}$ behaviour for the pion form factor as is predicted by QCD [21].

With a quark-photon vertex that satisfies the above constraints and using the identities:

$$
\begin{aligned}
S(-k)^{T} & =C^{T} S(k) C, \\
\bar{\Gamma}_{\pi}^{T}(-k ;-p) & =-C^{T} \Gamma_{\pi}(k ; p) C, \\
\Gamma_{\mu}^{T}(-k,-p) & =-C^{T} \Gamma_{\mu}(p, k) C,
\end{aligned}
$$

where $C=\gamma_{2} \gamma_{4}$ is the charge conjugation matrix, it is easy to establish that

$$
(p+q)_{\mu} \Lambda_{\mu}^{\mathrm{GIA}}(p, q)=0
$$


i.e., the $\pi$-current is conserved. Hence one can write

$$
\Lambda_{\mu}^{\mathrm{GIA}}(p, q)=T_{\mu}(p, q) \hat{F}_{\pi}^{\mathrm{GIA}}\left(p^{2}, p \cdot q, q^{2}\right)
$$

where

$$
T_{\mu}(p, q)=2 \frac{p_{\mu} q \cdot(p+q)-q_{\mu} p \cdot(p+q)}{(p+q)^{2}} .
$$

In the case of elastic scattering $p^{2}=q^{2}$ and therefore Eq. (28) reduces to

$$
\Lambda_{\mu}^{\mathrm{GIA}}(p, q)=(p-q)_{\mu} F_{\pi}^{\mathrm{GIA}}\left((p+q)^{2}, p^{2}\right) .
$$

Using Eqs. (24-26) one can also show that $\Lambda_{\mu}^{\mathrm{GIA}}(P,-P)$ is

$$
\begin{aligned}
2 P_{\mu} F^{\mathrm{GIA}}\left(0, P^{2}\right)=\frac{N_{c}}{f_{\pi}^{2}} \int \frac{d^{4} k}{(2 \pi)^{4}} \operatorname{tr}_{D} & {\left[\bar{\Gamma}_{\pi}(k ; P) S\left(k_{-0}\right) \Gamma_{\pi}(k ;-P) \frac{\partial}{\partial P_{\mu}} S\left(k_{+0}\right)\right.} \\
+ & \left.\bar{\Gamma}_{\pi}(k ; P) \frac{\partial}{\partial P_{\mu}} S\left(k_{-0}\right) \Gamma_{\pi}(k ;-P) S\left(k_{+0}\right)\right] .
\end{aligned}
$$

Comparing this with Eqs. (17) and (18) one observes that in generalised impulse approximation $F^{\mathrm{GIA}}\left(0, P^{2}\right)=1$ only if the Bethe-Salpeter kernel is independent of $P$; i.e., generalised impulse approximation combined with a $P$-independent Bethe-Salpeter kernel provides a consistent approximation scheme. In this case one has, in the chiral limit $P^{2}=-m_{\pi}^{2}=0$ :

$$
\begin{aligned}
& f_{\pi}^{2}= \\
& \frac{N_{c}}{8 \pi^{2}} \int_{0}^{\infty} d s s \Gamma_{\pi}(s)^{2}\left(\sigma_{V}^{2}-2\left[\sigma_{S} \sigma_{S}^{\prime}+s \sigma_{V} \sigma_{V}^{\prime}\right]-s\left[\sigma_{S} \sigma_{S}^{\prime \prime}-\left(\sigma_{S}^{\prime}\right)^{2}\right]-s^{2}\left[\sigma_{V} \sigma_{V}^{\prime \prime}-\left(\sigma_{V}^{\prime}\right)^{2}\right]\right) .
\end{aligned}
$$

This illustrates that Eq. (1) is regular in the chiral limit and only weakly dependent on $m_{\pi}$.

We are now in a position to consider the Ansatz for the quark propagator, Eqs. (7) and (8), in more detail. Following Ref. [17], this Ansatz for the fermion propagator can be used to fit the characteristic parameters of the $\pi-\pi$ sector with the result that, with

$$
\Lambda_{1}=0.527 \mathrm{GeV} \text { and } \Lambda_{2}=0.573 \mathrm{GeV} \text {, }
$$

one obtains

$$
f_{\pi}=0.093 \mathrm{GeV}(0.093), \quad r_{\pi}=0.53 \mathrm{fm}(0.66)
$$

and the following values for the dimensionless scattering lengths:

$$
\begin{array}{ll}
a_{0}^{0}=0.16(0.20), & a_{0}^{2}=-0.047(-0.037), \\
a_{1}^{1}=0.029(0.038), & a_{2}^{0}=0.0014(0.0017), \quad a_{2}^{2}=-0.00037,
\end{array}
$$

where the experimental values, when known, are given in parentheses. These results were obtained using the approximation of Eq. (15) and $f_{\pi}$ was calculated using Eq. (32). The formulae for the remaining quantites are all given in Ref. [17] and, apart from $\left(r_{\pi}^{2}\right)^{\text {GIA }}$ in Eq. (A1), will not be reproduced [36], however, all of the information needed to derive them is contained herein. The results are simply presented so as to demonstrate the efficacy and utility of this simple Ansatz and to fix reasonable values of the parameters. 


\section{Approximate form of $F^{\mathrm{GIA}}\left(p^{2}, p \cdot q, q^{2}\right)$}

All of the elements of the calculation of $F^{\mathrm{GIA}}\left(p^{2}, p \cdot q, q^{2}\right)$ have now been described. The actual calculation involves a three-dimensional integral that can be evaluated numerically without difficulty for arbitrarily large spacelike momentum transfer provided a sensible DSEBSE framework is used to describe the quark-gluon substructure of the pion. This is the subject of Ref. [21]. (The fact that Eqs. (7) and (8) should only be used for interpolation is important in this context.) Herein we are interested in the charge radius as it is affected by this parton substructure and by meson self-dressing. The detailed structure of $F^{\mathrm{GIA}}\left(p^{2}, p \cdot q, q^{2}\right)$ is not necessary for this calculation.

In general $F^{\text {GIA }}\left(p^{2}, p \cdot q, q^{2}\right)$ depends on all three of its arguments and, as will become clear below, this dependence will be sampled by the pion loop contribution to the charge radius. However, our calculation is greatly simplified if we make the approximation that

$$
F^{\mathrm{GIA}}\left(p^{2}, p \cdot q, q^{2}\right)=F^{\mathrm{GIA}}\left(Q^{2}\right)
$$

where $Q^{2}=(p+q)^{2}$, for then this piece appears only as a multiplicative factor in the pion loop contribution to the charge radius. This approximation is expected to lead to an overestimate of the importance of the pion loop to the charge radius since any off-shell effects in $F^{\text {GIA }}\left(p^{2}, p \cdot q, q^{2}\right)$ will lead to additional damping in the pion-loop integral, which is over spacelike momenta.

Subject to this approximation then, for our purposes, all we need to know is

$$
\left(r_{\pi}^{2}\right)^{\mathrm{GIA}}=-\left.6 \frac{d}{d Q^{2}} \ln F^{\mathrm{GIA}}\left(Q^{2}\right)\right|_{Q^{2}=0}
$$

which has been presented in Ref. [17] and which we reproduce in Eq. (A1) of the appendix. As we have seen, the simple interpolating form for the quark propagator that we are using herein yields the charge radius in Eq. (34) while the more sophisticated analysis of Ref. [17], using a somewhat different form of the fermion propagator, obtained the result $r_{\pi}=0.59 \mathrm{fm}$. Both of these calculations are to be compared with the experimental value of $r_{\pi}=0.66 \mathrm{fm}$ [34. This illustrates what we believe to be a general result: that the generalised impulse approximation consistently understimates the charge radius by $<15 \%$ leaving room for only small contributions from meson-dressing effects such as the pion loop and $\rho^{0}$-photon mixing.

\section{PION LOOP CONTRIBUTION}

We approximate the pion loop contribution to the connected $\pi \pi \gamma$ vertex as

$$
\begin{aligned}
& \Lambda_{\mu}^{i j \operatorname{Loop}}(p, q)= \\
& -\frac{1}{2} \epsilon^{3 k l} \int \frac{d^{4} k}{(2 \pi)^{4}} T^{i j k l}(p+q, p-k, k-q) D_{\pi}(k) D_{\pi}(p+q-k) \Lambda_{\mu}^{\mathrm{GIA}}(k, p+q-k)
\end{aligned}
$$

where $D_{\pi}(k)$ is the pion propagator, $\Lambda_{\mu}^{\mathrm{GIA}}(p, q)$ is given in Eq. (11) and the $\pi-\pi$ scattering kernel is $T^{i j k l}\left(p_{1}+p_{2}, p_{1}+p_{3}, p_{1}+p_{4}\right)$, which can be written in terms of a single scalar function: 


$$
\begin{aligned}
& T^{i j k l}\left(p_{1}+p_{2}, p_{1}+p_{3}, p_{1}+p_{4}\right)= \\
& \delta^{i j} \delta^{k l} A\left(p_{1}, p_{2} ; p_{3}, p_{4}\right)+\delta^{i k} \delta^{j l} A\left(p_{1}, p_{3} ; p_{2}, p_{4}\right)+\delta^{i l} \delta^{j k} A\left(p_{1}, p_{4} ; p_{3}, p_{2}\right)
\end{aligned}
$$

with

$$
A\left(p_{1}, p_{2} ; p_{3}, p_{4}\right)=A\left(p_{2}, p_{1} ; p_{3}, p_{4}\right)=A\left(p_{1}, p_{2} ; p_{4}, p_{3}\right)=A\left(p_{2}, p_{1} ; p_{4}, p_{3}\right)
$$

from which all of the relations of crossing symmetry follow. We also note that the result

$$
(p+q)_{\mu} \Lambda_{\mu}^{\mathrm{Loop}}(p, q)=0
$$

follows from Eq. (27) and as a consequence one may write

$$
\Lambda_{\mu}^{i j \operatorname{Loop}}(p, q)=\epsilon^{3 i j} T_{\mu}(p, q) \hat{F}_{\pi}^{\mathrm{Loop}}\left(p^{2}, p \cdot q, q^{2}\right)
$$

which, in the case of elastic scattering $\left[p^{2}=q^{2}\right]$, reduces to

$$
\Lambda_{\mu}^{i j \operatorname{Loop}}(p, q)=\epsilon^{3 i j}(p-q)_{\mu} F_{\pi}^{\mathrm{Loop}}\left((p+q)^{2}, p^{2}\right) .
$$

Equation (38) is illustrated in Fig. 2. This is the analogue in the GCM [and coupled DSEBSE approach] of the one pion loop diagram calculated in ChPT.

\section{A. $\pi-\pi$ scattering amplitude}

The $\pi-\pi$ scattering amplitude is an important part of Eq. (38). The form of $A\left(p_{1}, p_{2} ; p_{3}, p_{4}\right)$ near threshold in the GCM was studied in detail in Ref. [17. Therein it was shown to reproduce the Weinberg term [35] at $\mathrm{O}\left(\mathrm{E}^{2}\right)$ and to possess additional structure at $\mathrm{O}\left(\mathrm{E}^{4}\right)$ that provides for a better description of the scattering lengths and partial wave amplitudes in $\pi-\pi$ scattering:

$$
\begin{aligned}
A(s, t, u)= & \frac{m_{\pi}^{2}+2 s-t-u}{3 f_{\pi}^{2}} \\
+ & \frac{4 N_{c}}{3 f_{\pi}^{4}}\left[K_{1}\left(-12 m_{\pi}^{4}+6 m_{\pi}^{2}(s+t+u)+2 s^{2}-t^{2}-u^{2}-2(s t+s u+t u)\right)\right. \\
& \left.+K_{2}\left(-2 m_{\pi}^{2}+s\right)\left(-2 m_{\pi}^{2}+t+u\right)+K_{3}\left(-2 m_{\pi}^{4}+m_{\pi}^{2}(s+t+u)-t u\right)\right],
\end{aligned}
$$

where $s=-\left(p_{1}+p_{2}\right)^{2}, t=-\left(p_{1}+p_{3}\right)^{2}$ and $u=-\left(p_{1}+p_{4}\right)^{2}$ are the usual Mandelstam variables and in the GCM [17]: $K_{1}=0.000508, K_{2}=0.00930$ and $K_{3}=0.00101$.

In the present study, however, an expansion in powers of momenta about threshold is inadequate since the integration in Eq. (38) samples momenta well away from threshold. A broad ranging discussion of the constraints that analyticity and unitarity place on the asymptotic behaviour of the scattering amplitude can be found in Refs. [37]; one result being that the forward scattering amplitude, $A\left(s, 0,4 m_{\pi}^{2}-s\right)$, cannot fall faster than $s^{-2}$.

The dominant contribution to $A\left(p_{1}, p_{2} ; p_{3}, p_{4}\right)$ in the GCM is obtained from Eqs. (A.14) and (A.16b) in Ref. [16]:

$$
\int d^{4} x d^{4} y \frac{f_{\pi}^{2}}{2} \operatorname{tr}\left[\partial_{\mu} U(x) \partial_{\mu} U(y)^{\dagger}\right] \hat{f}(x-y)
$$


where, as usual, $U(x)=\exp \left(i \boldsymbol{\pi} \cdot \boldsymbol{\tau} / f_{\pi}\right)$ and

$$
\hat{f}(x)=\int \frac{d^{4} k}{(2 \pi)^{4}} \mathrm{e}^{i k \cdot x} f\left(k^{2}\right)
$$

with

$$
f_{\pi}^{2} p^{2} f(p)=2 N_{c} \int \frac{d^{4} k}{(2 \pi)^{4}} \Gamma_{\pi}\left(k^{2}\right)^{2} K\left(k_{+}\right) K\left(k_{-}\right)\left(k^{2} A_{-}^{2}+k \cdot p A_{-} A_{+}+\frac{1}{4} p^{2} A_{+}^{2}\right),
$$

$\left[k_{ \pm}=k \pm \frac{1}{2} p\right],\left[A_{ \pm}=A\left(k_{+}\right) \pm A\left(k_{-}\right)\right]$and where

$$
K(p)=\frac{1}{p^{2} A\left(p^{2}\right)^{2}+B\left(p^{2}\right)^{2}} .
$$

[This expression has been generalised here to allow for momentum dependence of $A\left(p^{2}\right)$.] In Eq. (47), the right-hand-side contributes the leading term in the expression for $f_{\pi}^{2}$ in the chiral limit: the other terms that contribute to Eq. (32) are obtained from Eqs. (A.16a) and (A.16c) in Ref. [16] and in the point-meson [or Nambu-Jona-Lasinio model] limit of the GCM, defined such that $A\left(p^{2}\right)=1$ and $B\left(p^{2}\right)=$ constant, these terms vanish. Taking this into account we renormalise Eq. (47) and define

$$
p^{2} f(p)=\frac{1}{\mathcal{N}^{\prime}} \int \frac{d^{4} k}{2 \pi^{2}} \Gamma_{\pi}\left(k^{2}\right)^{2} K\left(k_{+}\right) K\left(k_{-}\right)\left(k^{2} A_{-}^{2}+k \cdot p A_{-} A_{+}+\frac{1}{4} p^{2} A_{+}^{2}\right)
$$

with $\mathcal{N}^{\prime}$ chosen so that $f\left(p^{2}=0\right)=1$.

The contribution of Eq. (45) to $A\left(p_{1}, p_{2} ; p_{3}, p_{4}\right)$ at tree level is easily found to be

$$
A^{\mathrm{LO}}\left(p_{1}, p_{2} ; p_{3}, p_{4}\right)=\frac{1}{3 f_{\pi}^{2}}\left(3\left(p_{1}+p_{2}\right)^{2} f\left(p_{1}+p_{2}\right)-\sum_{i=1}^{4} p_{i}^{2} f\left(p_{i}^{2}\right)\right) \text {. }
$$

We refer to this expression as the "leading-order" scattering amplitude because it provides the leading contribution at large $s$; i.e., it is the contribution to the scattering amplitude in the GCM which falls least rapidly with increasing $s$. We note that neglecting the momentum dependence of $f(p)$; i.e., at lowest order in a momentum expansion, Eq. (50) yields, upon continuation to Minkowski space,

$$
A^{\mathrm{LO}}\left(p_{1}, p_{2} ; p_{3}, p_{4}\right)=\frac{2 s-t-u}{3 f_{\pi}^{2}}
$$

which reproduces the result of Ref. [35] in the chiral limit, $m_{\pi} \rightarrow 0$.

We would like an accurate estimate of this amplitude in the GCM. It is not difficult to evaluate the integral numerically using the results of DSE studies, as represented by Eqs. (7), (8) and (33), but it is more instructive to distill these ingredients and obtain an analytic expression that manifests the important features. To do this we use Eqs. (7), (8) and (15) but, in addition, we make a further simplifying assumption, based on the "constituent mass" concept described in Ref. [38]. The basic observation in this connection is that the integration in Eq. (47) is weighted, and cut off, by the factor $k^{2} \Gamma_{\pi}\left(k^{2}\right)^{2}$ that arises because of the quark-gluon substructure of the pion in the GCM. This manifestation of the quark core of the meson is a general feature of the coupled DSE-BSE approach and ensures that 
even meson loop integrals are intrinsically finite. This factor concentrates the integration domain around $s=\Lambda_{2}^{2}$, using Eq. (15), and hence it is a reasonable approximation to replace $B\left(p^{2}\right)$ in $K\left(p^{2}\right)$ by an "effective constituent quark mass":

$$
K\left(p^{2}\right)=\frac{1}{p^{2}+M_{c}^{2}}
$$

where a first estimate of $M_{c}$ is obtained by evaluating $B\left(p^{2}\right)$ at $p^{2}=\Lambda_{2}^{2}$, which is the maximum of $k^{2} \Gamma_{\pi}\left(k^{2}\right)^{2}$ :

$$
M_{c} \approx B\left(\Lambda_{2}^{2}\right)=\frac{\Lambda_{1}^{3}}{2 \Lambda_{2}^{2}} .
$$

Using the values of $\Lambda_{1}$ and $\Lambda_{2}$ in Eq. (33) this yields $M_{c} \sim 0.22 \mathrm{GeV}$.

A more sophisticated estimate of $M_{c}$ may be obtained by requiring that using Eq. (52) in Eq. (32) should yield the same result for $f_{\pi}$ as the exact calculation. Using the approximations of Eqs.(7), (8), (15) one can rewrite Eq. (32) as

$$
f_{\pi}^{2}=\frac{N_{c}}{8 \pi^{2}} \int_{0}^{\infty} d s s K(s)^{2} B(s)\left(B(s)-\frac{1}{2} B^{\prime}(s)\right),
$$

which, not unexpectedly, is the result presented in Ref. [24]. Substituting Eq. (52) for $K$ in this expression one obtains

$$
f_{\pi}^{2}=\frac{N_{c} \Lambda_{1}^{6}}{16 \pi^{2}} \frac{\left(-7 \Lambda_{2}^{4}+20 \Lambda_{2}^{2} M_{c}^{2}-13 M_{c}^{4}+\ln \frac{M_{c}^{2}}{\Lambda_{2}^{2}}\left[-4 \Lambda_{2}^{4}+4 \Lambda_{2}^{2} M_{c}^{2}+6 M_{c}^{4}\right]\right)}{\left(M_{c}^{2}-\Lambda_{2}^{2}\right)^{4}}
$$

and requiring that this yield $f_{\pi}=0.093 \mathrm{GeV}$ gives

$$
M_{c}=0.31 \mathrm{GeV},
$$

which shows that Eq. (53) provides a reasonable estimate.

Using Eqs. (7), (8), (15) and (52) we obtain

$$
p^{2} f(p)=\frac{2}{\mathcal{N}} \int_{0}^{\infty} d u \frac{1}{\left(u+\Lambda_{2}^{2}\right)^{2}}\left(1-\frac{\sqrt{\left(u+\frac{1}{4} p^{2}+M_{c}^{2}\right)^{2}-u p^{2}}}{u+\frac{1}{4} p^{2}+M_{c}^{2}}\right)
$$

with

$$
\mathcal{N}=\frac{\left(M_{c}^{2}+\Lambda_{2}^{2}\right) \ln \frac{M_{c}^{2}}{\Lambda_{2}^{2}}-2\left(M_{c}^{2}-\Lambda_{2}^{2}\right)}{\left(M_{c}^{2}-\Lambda_{2}^{2}\right)^{3}}
$$

It is not difficult to establish that, at large- $p^{2}$,

$$
f(p) \approx \frac{1}{\mathcal{N}} \frac{8}{p^{4}} \ln \frac{p^{2}}{\Lambda_{2}^{2}}
$$

which gives $A^{\mathrm{LO}}(s, t, u)$ consistent with the bounds of Refs. [37]. We emphasise that $\Lambda_{2}$ is not an arbitrary parameter introduced to regularise the integral but, rather, it is a direct measure of the quark-gluon substructure in the model arising as it does through solving the Bethe-Salpeter equation; i.e., it is a calculated quantity in the GCM. We note also that $\Lambda_{1}$ has not disappeared but is contained implicitly in $M_{c}$. 


\section{NET $\pi \pi \gamma$ VERTEX}

The net $\pi \pi \gamma$ vertex is obtained by adding Eq. (38) directly to Eq. (11):

$$
\begin{aligned}
\Lambda_{\mu}^{i j}(p, q) & =\epsilon^{3 i j} \Lambda_{\mu}^{\mathrm{GIA}}(p, q)+\Lambda_{\mu}^{i j \operatorname{Loop}}(p, q) \\
& \equiv \epsilon^{3 i j} T_{\mu}(p, q) \hat{F}_{\pi}\left(p^{2}, p \cdot q, q^{2}\right)
\end{aligned}
$$

with

$$
\hat{F}_{\pi}\left(p^{2}, p \cdot q, q^{2}\right)=\hat{F}_{\pi}^{\mathrm{GIA}}\left(p^{2}, p \cdot q, q^{2}\right)+\hat{F}_{\pi}^{\mathrm{Loop}}\left(p^{2}, p \cdot q, q^{2}\right) .
$$

This does not lead to double-counting because the pion final-state-interactions cannot be recast into an additional dressing of the quark-photon vertex; unlike the $\rho$-meson contribution that is contained in $\Gamma^{T}(p, k)$ and which we neglected. Adding Eq. (38) is thus a correction beyond generalised impulse approximation and as such leads to a renormalisation of the pion field in our approach, which is simply a reordering of the summation of diagrams in a well defined and systematic manner as prescribed by the bosonisation of the GCM.

To clarify this statement we remark that going beyond generalised impulse approximation in the calculation of the electromagnetic pion form factor builds additional structure into the pion. In the framework of the GCM, the "bare pion"; i.e., the one whose Bethe-Salpeter amplitude appears in Eq. (11), is a ladder $q-\bar{q}$ bound state: it represents the "quark core" of the pion. As shown in Ref. [17], this core provides a very good description of kinematic and dynamical properties of the pion away from resonance contributions. Adding the pion loop contribution is the first step in allowing this ladder $q-\bar{q}$ bound state to dress itself with a cloud of ladder mesonic bound states. In the framework of the BSE, Eq. (9), this corresponds to going beyond ladder approximation. Indeed, this modification could be built into the pion BSE and would lead to a dependence on the centre-of-mass momentum, $P$, in the kernel of Eq. (9) thus changing the normalisation obtained from Eq. (17). This illustrates the nature of the GCM approach. The "tree-level" fields in the effective action represent ladder $q-\bar{q}$ bound states and the remaining interaction terms provide for meson self-dressing, in addition to meson-meson interactions, which extends the nature of the structure of the bound state beyond that of ladder approximation.

The pion propagator in Eq. (38) has the form

$$
D_{\pi}(k)=\frac{1}{k^{2}+\Sigma_{\pi}^{2}\left(k^{2}\right)}
$$

where $\Sigma_{\pi}^{2}\left(k^{2}\right)$ is the pion self energy which can be obtained as the solution of a DSE or using the eigen-value procedure of Ref. [18]. Herein we follow Ref. [39] and use the approximation

$$
D_{\pi}(k)=\frac{1}{k^{2}+\left(m_{\pi}^{\mathrm{L}}\right)^{2}},
$$

where $\left(m_{\pi}^{\mathrm{L}}\right)^{2}-\Sigma_{\pi}^{2}\left(-\left(m_{\pi}^{\mathrm{L}}\right)^{2}\right)=0$. We have labelled the mass of the pion in the loop $m_{\pi}^{\mathrm{L}}$ in order to artificially distinguish it from the mass of the external pions; a convention we adopt simply so as to make explicit the dependence of the charge radius on the mass of the loop particle. Equation (64) is expected to be a good approximation because the Bethe-Salpeter 
amplitudes in Eqs. (1) and (38) ensure that the dominant integration domain is at small $k^{2}$ where the pion propagator should not be much modified from its on-shell form. Indeed, the dominant dressing contributions for the virtual pion propagator would come from a $3 \pi$ intermediate state and this was shown to be negligible in Ref. [39.

Using Eq. (64) in Eq. (62) we obtain the following general expression for the one-pionloop corrected approximation to the electromagnetic pion form factor:

$$
\begin{aligned}
& \hat{F}_{\pi}\left(p^{2}, p \cdot q, q^{2}\right)=\hat{F}_{\pi}^{\mathrm{GIA}}\left(p^{2}, p \cdot q, q^{2}\right) \\
& -\frac{1}{2} \int \frac{d^{4} k}{(2 \pi)^{4}}\left[\frac{q \cdot(p+q) k \cdot(p+q)-q \cdot k(p+q)^{2}}{p^{2} q^{2}-(p \cdot q)^{2}} \times\right. \\
& \left.\frac{A(p,-k ; q, k-p-q)-A(p, k-p-q ; q,-k)}{\left(k^{2}+\left(m_{\pi}^{\mathrm{L}}\right)^{2}\right)\left((-k+p+q)^{2}+\left(m_{\pi}^{\mathrm{L}}\right)^{2}\right)} \hat{F}_{\pi}^{\mathrm{GIA}}\left(k^{2}, k \cdot(-k+p+q),(-k+p+q)^{2}\right)\right] .
\end{aligned}
$$

As we have mentioned above and make explicit below, the pion loop contribution is given

by a finite integral because the "bare meson" in the loop already has an internal structure: its $q-\bar{q}$ core as given by the BSE, Sec. IIB.

\section{PION CHARGE RADIUS}

Equation (65) is simplified by the approximations we have described above. Using Eq. (36), the "leading order" scattering amplitude, Eqs. (50) and (57), shifting the integration variable: $[k \rightarrow k+q]$, writing

$$
P=p-q \text { and } Q=p+q,
$$

introducing hyperspherical polar coordinates and placing the external pions on the chirallimit mass-shell, $\left[P^{2}+Q^{2}=0\right]$, we obtain the following expression 40]:

$$
\begin{aligned}
\hat{F}_{\pi}\left(Q^{2}\right)= & \hat{F}_{\pi}^{\mathrm{GIA}}\left(Q^{2}\right)\left\{1+\frac{1}{16 \pi^{3} f_{\pi}^{2}}\left[\int_{0}^{\infty} d k k^{3} \int_{-1}^{1} d y\left(1+2 i y \frac{k}{Q}\right)\right.\right. \\
& \times \frac{\left(k^{2}-2 i Q k y-Q^{2}\right) f\left(k^{2}-2 i Q k y-Q^{2}\right)-k^{2} f\left(k^{2}\right)}{k Q\left(k^{2}-i k Q y+\left(m_{\pi}^{\mathrm{L}}\right)^{2}\right)} \\
& \left.\left.\times \ln \left|\frac{k^{2}-i k Q y+\left(m_{\pi}^{\mathrm{L}}\right)^{2}+k Q \sqrt{1-y^{2}}}{k^{2}-i k Q y+\left(m_{\pi}^{\mathrm{L}}\right)^{2}-k Q \sqrt{1-y^{2}}}\right|\right]\right\}
\end{aligned}
$$

Equation (67) can be used to calculate the one-pion-loop corrected value of the pion decay constant, which we will denote $\hat{f}_{\pi}$,

$$
\hat{f}_{\pi}^{2}=f_{\pi}^{2} \hat{F}_{\pi}(0)
$$

A little algebra yields

$$
\hat{f}_{\pi}^{2}-f_{\pi}^{2}=\frac{2}{\pi^{2}} \int_{0}^{\infty} d u \frac{u^{2}}{\left[4 u+\left(m_{\pi}^{\mathrm{L}}\right)^{2}\right]^{2}} g(u)
$$

where, with $f(x)$ given in Eq. (57), 


$$
\begin{aligned}
g(u) & =f(4 u)+u \frac{d}{d u} f(4 u) \\
& =\frac{1}{\mathcal{N}} \int_{0}^{\infty} d v \frac{v\left(v+M_{c}^{2}-u\right)}{\left(v+\Lambda_{2}^{2}\right)^{2}\left(v+M_{c}^{2}+u\right)^{2} \sqrt{\left(v+M_{c}^{2}+u\right)^{2}-4 u v}}
\end{aligned}
$$

and $\mathcal{N}$ is given in Eq. (58). This integral can be evaluated and the result is given in Eq. (A3) of the appendix.

The result in Eq. (34) and the more detailed study of Ref. [17], which obtained $f_{\pi}=0.091 \mathrm{GeV}$, suggest that meson-loop effects should not make a large contribution to the normalisation. This is indeed what we find, as illustrated in Fig. 3 which shows that for reasonable values of $M_{c}$ and $\Lambda_{2}$ :

$$
0.2 \mathrm{GeV}<M_{c}<0.6 \mathrm{GeV} \text { and } 0.3 \mathrm{GeV}<\Lambda_{2}<0.7 \mathrm{GeV}
$$

the correction is small and negative, leading to a less than $2 \%$ correction at the physical value of the pion mass.

The relative importance of the quark core and pion-loop contributions to the electromagnetic pion charge radius, $r_{\pi}$, can also be obtained from Eq. (67):

$$
\hat{r}_{\pi}^{2}=-\left.6 \frac{d}{d Q^{2}} \ln \hat{F}_{\pi}\left(Q^{2}\right)\right|_{Q^{2}=0}
$$

Substituting we obtain

$$
\hat{r}_{\pi}^{2}=\left(r_{\pi}^{2}\right)^{\mathrm{GIA}}+\frac{16}{\pi^{2} \hat{f}_{\pi}^{2}} \int_{0}^{\infty} d u \frac{u^{3}}{\left[4 u+\left(m_{\pi}^{\mathrm{L}}\right)^{2}\right]^{4}} g(u)
$$

where $\left(r_{\pi}^{2}\right)^{\text {GIA }}$ is the generalised impulse approximation contribution.

The result in Eq. (34) and that of $r_{\pi}=0.59$ fm obtained in Ref. [17] suggest that mesonloop effects can additively contribute $10-15 \%$ to the charge radius at the physical value of the pion mass. The same conclusion can be drawn from the phenomenological Coulomb gauge DSE studies of Ref. [41]. This is just what we find, as we illustrate in Fig. [t which shows that with $\hat{f}_{\pi}=0.093 \mathrm{GeV}$, reasonable values of $M_{c}$ and $\Lambda_{2}$, Eq. (72), and at the physical value of the pion mass, the correction to the square of the charge radius is always positive and less than $15 \%$.

It is of interest to study the chiral limit. It will be recalled that we have set the mass of all the external pions to zero so that the contribution of the loop-pions in the chiral limit is easily identified: $m_{\pi}^{\mathrm{L}} \rightarrow 0$. As we have remarked, the generalised impulse approximation contribution, $\left(r_{\pi}^{\mathrm{GIA}}\right)$, is regular in the chiral limit and only weakly dependent on the current quark mass. In Fig. 5 we plot the ratio $\hat{r}_{\pi}^{2} /\left(r_{\pi}^{\mathrm{GIA}}\right)^{2}$ as a function of $m_{\pi}^{\mathrm{L}}$ for $M_{c}=0.22 \mathrm{GeV}$ and $\Lambda_{2}=0.5 \mathrm{GeV}$. This figure emphasises that at $m_{\pi}^{\mathrm{L}} \approx 0.14 \mathrm{GeV}$ the dominant contribution to the pion charge radius is provided by the quark core. It is not until $m_{\pi}^{\mathrm{L}}$ becomes very small, on the order of $0.01 \mathrm{GeV}$, that the pion cloud contribution becomes as important as the quark core and this contribution is well described by the form

$$
\left(r_{\pi}^{2}\right)^{\operatorname{div}}=\left(r_{\pi}^{2}\right)^{\mathrm{GIA}}\left[0.73-0.082 \ln \left(\frac{\left(m_{\pi}^{\mathrm{L}}\right)^{2}}{m_{\rho}^{2}}\right)\right] .
$$


for $m_{\pi}^{\mathrm{L}}<0.14 \mathrm{GeV}$.

It is natural to compare our result with that of ChPT. In Ref. [四] one finds in Eq. (5.6) the result

$$
\left\langle r^{2}\right\rangle_{\pi}=\frac{12 L_{9}^{r}}{F_{0}^{2}}-\frac{1}{32 \pi^{2} F_{0}^{2}}\left(2 \ln \left[\frac{m_{\pi}^{2}}{\mu^{2}}\right]+\ln \left[\frac{m_{\kappa}^{2}}{\mu^{2}}\right]+3\right)
$$

where $\mu^{2}$ is the loop regularisation scale and $L_{9}^{r}$ is one of the ten standard low energy constants in the effective action of ChPT into which an infinity from the divergence of the pseudoscalar loop has been absorbed [42].

The ten low energy coefficients, of which $L_{9}^{r}$ is one, are fixed by requiring that the effective action of ChPT provide a good description of low energy strong interaction phenomena while the loop regularisation scale $\mu^{2}$ is usually taken to be something of the order of $m_{\eta}^{2}$ or $m_{\rho}^{2}$ because this is a measure of the mass scale at which additional excitations should be accounted for. Of course, the actual values of the coefficients $L_{i}^{r}$ depend on $\mu^{2}$ because of the ambiguities associated with regularising the divergent loops but, once a scale is chosen, values can be quoted. of

Fitting $\kappa_{e 3}^{0}$ decay using $\mu^{2}=m_{\eta}^{2}$ [Ref. [3], below Eq. (14.1)] and $F_{0}=93.3 \mathrm{MeV}$ a value

$$
L_{9}^{r}=(7.1 \pm 0.4) \times 10^{-3}
$$

is quoted in Ref. [4] in which case

$$
\frac{12 L_{9}^{r}}{F_{0}^{2}}=(0.38 \pm 0.020) \mathrm{fm}^{2},
$$

which is $90 \%$ of the experimental value: $0.439 \pm 0.008 \mathrm{fm}^{2}$ [34]. A similar calculation in Ref. 43 using $\mu^{2}=m_{\rho}^{2}$ with

$$
L_{9}^{r}=(6.9 \pm 0.2) \times 10^{-3}
$$

yields [upon correction of a minor typographical error therein]

$$
\frac{12 L_{9}^{r}}{F_{0}^{2}}=(0.37 \pm 0.011) \mathrm{fm}^{2},
$$

which is $84 \%$ of the experimental value. One sees that at the physical value of the pion mass, and with an accepted value of the renormalisation scale, the "chiral logarithm" contributes little to the charge radius of the pion.

The pion charge radius has also been studied in nonlinear chiral theories, Ref. [1], where a comparison is made between the contribution of nucleon and pion loops. In this study the pion loop contribution at the physical value of the pion mass was directly identified and found to be $0.06 \mathrm{fm}^{2}$ which is just $14 \%$ of the experimental value. 


\section{SUMMARY AND CONCLUSIONS}

Herein we have calculated the charge radius of the pion in the coupled Dyson-SchwingerBethe-Salpeter equation approach as formalised in the Global Colour-symmetry model, which is a QCD based model field theory. In this approach, the "bare hadrons" are nonpointlike objects with a "quark core" obtained as the solution of Bethe-Salpeter (mesons) or relativistic Faddeev (baryons) equations whose kernel is chosen in such a way as to model the quark-quark interaction in QCD. The meson cloud associated with a given hadron is a small correction in this framework and is obtained through a systematic expansion based on the effective action of the GCM.

In this approach the charge radius of the pion receives a contribution from its quark core and from pion loops. We showed that the quark core contribution is finite in the chiral limit and that, at the physical value of the pion mass, it is the dominant determining characteristic of the pion with the pion loop contribution being a small, finite, additive correction of less than $15 \%$. The fact that the loop contribution is finite is a general property of this formalism; it is due to the internal quark core structure which provides a natural cutoff in all integrals that arise. Our result is consistent with a recent reanalysis of the pion charge radius obtained in lattice simulations 44 .

We explicitly identified the origin of the chiral divergence in the charge radius of the pion as arising from the pion loop; this feature is not absent in the general DSE-BSE approach but is simply an higher order correction, as are all meson loop effects. Our calculation demonstrated that the pion's own pion cloud is not a very significant component unless the mass of the pion is less than $\sim 50 \mathrm{MeV}$.

A broad implication of our result is that it demonstrates that the many quark-based models which do not reproduce the logarithmic divergence of the charge radius in the chiral limit; for example, the relativistic, constituent quark model of Ref. [45], nevertheless capture the most important characteristic of the pion: its quark core, and should not be discarded on this basis alone.

In closing we note that the contribution of the quark core of the proton/neutron to its charge radius has not been calculated in the approach we have used herein, although such a calculation is underway. In our opinion, and if the analysis of Ref. 444 is a reasonable guide, the pion loop contribution will be a little more important in this case but, at the physical pion mass, will still generate less than a $30 \%$ correction.

\section{ACKNOWLEDGMENTS}

RA and AB take this opportunity to express their gratitude to Prof. Dr. H. Reinhardt for his support and interest over the course of this work. In addition, AB would like to thank the Physics Division of Argonne National Laboratory for their hospitality and for financial support during a visit in which a significant part of this work was conducted; and also gratefully acknowledges financial support from the Studienstiftung des deutschen Volkes. CDR takes this opportunity to thank Prof. Dr. H. Reinhardt for his hospitality during two visits to Universität Tübingen: one in which this collaboration was formed and another in order to bring this work to a conclusion. The authors also gratefully acknowledge

useful discussions with F. Coester. The work of CDR was supported by the US Department 
of Energy, Nuclear Physics Division, under contract number W-31-109-ENG-38. Some of the calculations described herein were carried out using a grant of computer time and the resources of the National Energy Research Supercomputer Center.

\section{APPENDIX: MISCELLANEOUS EXPRESSIONS}

In this appendix we present expressions for $\left(r_{\pi}^{2}\right)^{\mathrm{GIA}}$, Eq. (A1), and $g(u)$, Eq. (A3), since they are rather lengthy.

The generalised impulse approximation contribution to the pion charge radius is [17]:

$$
\left(r_{\pi}^{2}\right)^{\mathrm{GIA}}=\frac{N_{c}}{2 f_{\pi}^{2}} \int \frac{d^{4} q}{(2 \pi)^{4}} H\left(q^{2}\right)
$$

where

$$
\begin{aligned}
H\left(x=q^{2}\right)= & B\left(-A^{2} B^{2} B^{\prime} K^{\prime \prime} K^{2} x^{2}-A^{4} B^{\prime \prime} K^{\prime} K^{2} x^{3}-36 A^{2} B^{3} K^{\prime} K^{2}\right. \\
& -A^{4} B^{\prime} K^{\prime \prime} K^{2} x^{3}+A^{2} B A^{\prime} A^{\prime \prime} K^{3} x^{3}-30 A B^{3} A^{\prime} K^{3} \\
& -42 A^{2} B^{2} B^{\prime} K^{3}-24 A^{2} B^{3} K^{\prime \prime} K^{2} x-48 A^{4} B K^{\prime} K^{2} x \\
& -54 A B^{3} A^{\prime} K^{\prime} K^{2} x-42 A^{2} B^{2} B^{\prime} K^{\prime} K^{2} x-72 A^{3} B A^{\prime} K^{3} x \\
& -18 B^{3} A^{\prime 2} K^{3} x-24 A^{4} B^{\prime} K^{3} x-42 A B^{2} A^{\prime} B^{3} x \\
& -24 A B^{3} A^{\prime \prime} K^{3} x-21 A^{2} B^{2} B^{\prime \prime} K^{3} x-4 A^{2} B^{3} K^{\prime 3} x^{2} \\
& +4 A^{2} B^{3} K^{\prime \prime} K^{\prime} K x^{2}-6 A B^{3} A^{\prime} K^{\prime 2} K x^{2}-2 A^{2} B^{2} B^{\prime} K^{2} K x^{2} \\
& -24 A^{4} B K^{\prime \prime} K^{2} x^{2}-3 A B^{3} A^{\prime} K^{\prime \prime} K^{2} x^{2}-68 A^{3} B A^{\prime} K^{\prime} K^{2} x^{2} \\
& -2 B^{3} A^{\prime 2} K^{\prime} K^{2} x^{2}-28 A^{4} B^{\prime} K^{\prime} K^{2} x^{2}-18 A B^{2} A^{\prime} B^{\prime} K^{\prime} K^{2} x^{2} \\
& -8 A B^{3} A^{\prime \prime} K^{\prime} K^{2} x^{2}+3 A^{2} B^{2} B^{\prime \prime} K^{\prime} K^{2} x^{2}-20 A^{2} B A^{\prime 2} K^{3} x^{2} \\
& -40 A^{3} A^{\prime} B^{\prime} K^{3} x^{2}-14 A B A^{\prime} B^{\prime 2} K^{3} x^{2}+2 A^{2} B^{3} K^{3} x^{2} \\
& -36 A^{3} B A^{\prime \prime} K^{3} x^{2}-5 B^{3} A^{\prime} A^{\prime \prime} K^{3} x^{2}-4 A B^{2} B^{\prime} A^{\prime \prime} K^{3} x^{2} \\
& -8 A^{4} B^{\prime \prime} K^{3} x^{2}+2 A B^{2} A^{\prime} B^{\prime \prime} K^{3} x^{2}+4 A^{2} B B^{\prime} B^{\prime \prime} K^{3} x^{2} \\
& -4 A^{4} B K^{\prime 3} x^{3}+4 A^{4} B K^{\prime \prime} K^{\prime} K x^{3}-6 A^{3} B A^{\prime} K^{\prime 2} K x^{3} \\
& -2 A^{4} B^{\prime} K^{\prime 2} K x^{3}-3 A^{3} B A^{\prime} K^{\prime \prime} K^{2} x^{3}-10 A^{2} B A^{\prime 2} K^{\prime} K^{2} x^{3} \\
& -10 A^{3} A^{\prime} B^{\prime} K^{\prime} K^{2} x^{3}-4 A^{3} B A^{\prime \prime} K^{\prime} K^{2} x^{3}-6 B^{\prime \prime} A^{3} K^{3} x^{3} \\
& \left.-6 A^{2} A^{\prime 2} B^{\prime} K^{3} x^{3}-2 A^{3} B^{\prime} A^{\prime \prime} K^{3} x^{3}-2 A^{3} A^{\prime} B^{\prime \prime} K^{3} x^{3}\right)
\end{aligned}
$$

As we remarked above, the integral in Eq. (71) can be evaluated. Writing $x=M_{c}^{2} / \Lambda_{2}^{2}$ and $\tilde{u}=u / \Lambda_{2}^{2}$ one obtains:

$$
\begin{aligned}
g(u)= & \frac{(x-1)^{3}}{((1+x) \ln x+2(1-x))(\tilde{u}-1+x)^{3}\left[(\tilde{u}-1+x)^{2}+4 \tilde{u}\right]} \times \\
& \left\{(\tilde{u}+1-x)(\tilde{u}-1+x)^{2}\right. \\
& +\frac{4 \tilde{u} x(\tilde{u}-1+x)+(\tilde{u}+1-x)(\tilde{u}+1+x)\left[(\tilde{u}-1+x)^{2}+4 \tilde{u}\right]}{\sqrt{(\tilde{u}-1+x)^{2}+4 \tilde{u}}}
\end{aligned}
$$




$$
\begin{aligned}
& \times \ln \left(\frac{\sqrt{(\tilde{u}-1+x)^{2}+4 \tilde{u}}-(\tilde{u}+1-x)}{(\tilde{u}+x) \sqrt{(\tilde{u}-1+x)^{2}+4 \tilde{u}}+(\tilde{u}+x)^{2}+\tilde{u}-x}\right) \\
& +\frac{[(\tilde{u}+1)(\tilde{u}-1+x)+(\tilde{u}+1-x)(\tilde{u}+1+x)]\left[(\tilde{u}-1+x)^{2}+4 \tilde{u}\right]}{2 \sqrt{\tilde{u}(\tilde{u}+x)}} \\
& \left.\times \ln \left(\frac{\sqrt{\tilde{u}(\tilde{u}+x)}+\tilde{u}}{\sqrt{\tilde{u}(\tilde{u}+x)}-\tilde{u}}\right)\right\} .
\end{aligned}
$$




\section{REFERENCES}

EMail alkofer@ptsun1.tphys.physik.uni-tuebingen.de, bender@ptsun1.tphys.physik.uni-tuebingen.de, cdroberts@anl.gov

[1] D. Ebert and M. K. Volkov, Fort. Phys. 29, 35 (1981).

[2] J. Gasser and H. Leutwyler, Ann. Phys. (NY) 158, 142 (1983).

[3] J. Gasser and H. Leutwyler, Nucl. Phys. B 250, 465 (1985).

[4] J. Gasser and H. Leutwyler, Nucl. Phys. B 250, 517 (1985).

[5] H. Leutwyler, Chiral Effective Lagrangians in Perspectives in the Standard Model, Editors: R. K. Ellis, C. T. Hill and J. D. Lykken (World Scientific, Singapore, 1992).

[6] S. Weinberg, Physica A 96, 327 (1979).

[7] A. Hädicke, Int. J. Mod. Phys. A 6, 3321 (1991).

[8] C. D. Roberts, Schwinger Dyson Equations: Dynamical Chiral Symmetry Breaking and Confinement, in Proceedings of "The Workshop on QCD Vacuum Structure", American University of Paris, 1-5 June 1992, Editors H. M. Fried and B. Müller (World Scientific, New York, 1993).

[9] C. D. Roberts and A. G. Williams, Dyson-Schwinger Equations and their Application to Hadronic Physics, to appear in Progress in Particle and Nuclear Physics, Edited by A. Fäßler (Pergamon Press, Oxford, 1994).

[10] R. Delbourgo and M. D. Scadron, J. Phys. G 5, 1631 (1979).

[11] J. Praschifka, R. T. Cahill and C. D. Roberts, Int. J. Mod. Phys. A. 4, 4929 (1989).

[12] K. -I. Aoki, T. Kugo and M. K. Mitchard, Phys. Lett. B 266, 467 (1991).

[13] P. Jain and H. Munczek, Phys. Rev. D 46, 438 (1992); P. Jain and H. Munczek, " $q-\bar{q}$ bound states in the Bethe-Salpeter formalism", U. Kansas preprint number KU-HEP93-30, (1993).

[14] S. J. Stainsby and R. T. Cahill, "Quark-Gluon Vertex and Structure of Mesons and Diquarks", Flinders University Preprint, (1993).

[15] R. T. Cahill and C. D. Roberts, Phys. Rev. D 32, 2419 (1985).

[16] C. D. Roberts, R. T. Cahill and J. Praschifka, Ann. Phys. (NY) 188, 20 (1988).

[17] C. D. Roberts, R. T. Cahill, M. E. Seviour and N. Iannella, " $\pi-\pi$ scattering in a QCD based model field theory", ANL Preprint No. ANL-PHY-7512-TH-93, to appear in Phys. Rev. D.

[18] R. T. Cahill, Nucl. Phys. A 543, 63 (1992).

[19] B. Holdom, J. Terning and K. Verbeek, Phys. Lett. B 245, 612 (1990).

[20] M. R. Frank and P. C. Tandy, "Gauge Invariance and the Electromagnetic Current of Composite Pions", CEBAF Preprint, No. CEBAF-TH-93-05, (1993). Accepted for publication in Phys. Rev. C.

[21] C. D. Roberts, in progress.

[22] D. Atkinson and D. W. E. Blatt, Nucl. Phys. B 151, 342 (1979); S. J. Stainsby and R. T. Cahill, Phys. Lett. A 146, 467 (1990); P. Maris and H. Holties, Int. J. Mod. Phys. A 7, 5369 (1992); S. J. Stainsby and R. T. Cahill, Int. J. Mod. Phys. A 7 (1992) 7541.

[23] C. J. Burden, C. D. Roberts and A. G. Williams, Phys. Lett. B285, 347 (1992).

[24] H. Pagels and S. Stokar, Phys. Rev. D 20, 2947 (1979).

[25] C. H. Llewellyn-Smith, Ann. Phys. (N.Y.) 53, 521 (1969).

[26] M. R. Frank, private communication. 
[27] D. C. Curtis and M. R. Pennington, Phys. Rev. D 42, 4165 (1990); D. C. Curtis and M. R. Pennington, Phys. Rev. D 44, 536 (1991); D. C. Curtis and M. R. Pennington, Phys. Rev. D 46, 2663 (1992).

[28] C. J. Burden, How can $Q E D_{3}$ help us understand $Q C D_{4}$, in Proceedings of "The Workshop on QCD Vacuum Structure", American University of Paris, 1-5 June 1992, Editors H. M. Fried and B. Müller (World Scientific, New York, 1993); C. J. Burden and C. D. Roberts, Phys. Rev. D 44, 540 (1991).

[29] C. J. Burden, J. Praschifka and C. D. Roberts, Phys. Rev. D 46, 2695 (1992).

[30] C. J. Burden and C. D. Roberts, Phys. Rev. D 47, 5581 (1993).

[31] J. S. Ball and T.-W. Chiu, Phys. Rev. D22, 2542 (1980).

[32] A. H. Blin, B. Hiller and M. Schaden, Z. Phys. A. 331, 75 (1988).

[33] V. Bernard and U. -G. Meißner, Phys. Rev. Lett. 61, 2296 (1988).

[34] S. R. Amendolia, et al, Nucl. Phys. B 277, 168 (1986).

[35] S. Weinberg, Phys. Rev. Lett. 17, 616 (1966).

[36] The relevant equations in Ref. [17] are (28),(29), (33)-(37) and (39).

[37] Y. S. Jin and A. Martin, Phys. Rev. 135, B1369 (1964); ibid, B1375 (1964).

[38] J. Praschifka, R. T. Cahill and C. D. Roberts, Mod. Phys. Lett. A. 3, 1595 (1988).

[39] L. C. L. Hollenberg, C. D. Roberts and B. H. J. McKellar, Phys. Rev. C 46, 2057 (1992).

[40] This expression only receives finite and small corrections proportional to the mass of the particles in the external legs.

[41] K. Langfeld, R. Alkofer and P. A. Amundsen, Z. Phys. C. 42, 159 (1989).

[42] One might make an operational identification of the $L_{9}^{r}$ term with the quark core contribution in our approach. This might be argued by interpreting the absorption of the infinity from the pion loop as a step towards properly representing the nonpointlike nature of the physical pseudoscalar mesons.

[43] U. -G. Meißner, Rep. Prog. Phys. 56, 903 (1993).

[44] D. B. Leinweber and T. D. Cohen, Phys. Rev. D. 47, 2147 (1993).

[45] P. L. Chung, F. Coester and W. N. Polyzou, Phys. Lett. B 205, 545 (1988). An analysis of the chiral limit of this calculation, defined operationally as the limit $M_{c} \rightarrow 0$, where $M_{c}$ is the consituent quark mass, was made available to us in private communication from F. Coester. Depending on the form of the momentum space quark wave function one finds that $\left\langle r_{\pi}^{2}\right\rangle$ diverges at least as fast as $1 / M_{c}$. It should be noted, however, that the "real" chiral limit corresponds to $m \rightarrow 0$, where $m$ is the current quark mass, and in the framework used herein, $M_{c}$ remains finite as $m \rightarrow 0$. 


\section{FIGURES}

FIG. 1. This figure is a pictorial representation of the amplitude identified with the $\pi \pi \gamma$ vertex in a generalised impulse approximation. The straight external lines represent the incoming and outgoing $\pi$, the filled circles at the $\pi$ legs represent the $\langle\pi \mid \bar{q} q\rangle$ Bethe-Salpeter amplitudes, the wiggly line represents the photon, $\gamma$, the shaded circle at the $\gamma$ leg represents the regular part of the dressed quark-photon vertex [which satisfies the Ward-Takahashi Identity, Eq. (19)] and the broken internal lines represent the dressed quark propagator.

FIG. 2. This figure is a pictorial representation of our approximation to the pion loop contribution to the $\pi \pi \gamma$ vertex, Eq. (38). The straight external lines represent the external pions, the large filled region at the $\pi$ legs represents $T^{i j k l}(p+q, p-k, k-q)$, Eq. (39), the internal lines represent the virtual loop pions, the smaller filled region represents $\Lambda_{\mu}^{\mathrm{GIA}}(k, p+q-k)$, Fig. 1, and the wiggly line represents the photon.

FIG. 3. In this figure we plot $\left[f_{\pi}-\hat{f}_{\pi}\right]$, in $\mathrm{MeV}$, in order to illustrate the dependence of the pion-loop induced renormalisation of $f_{\pi}$ on $M_{c}$ and $\Lambda_{2}$, physically reasonable ranges for which are $0.2 \mathrm{GeV}<M_{c}<0.6 \mathrm{GeV}$ and $0.3 \mathrm{GeV}<\Lambda_{2}<0.7 \mathrm{GeV}$.

FIG. 4. In this figure we plot $\left[\hat{r}_{\pi}^{2} /\left(r_{\pi}^{2}\right)^{\text {GIA }}-1\right]$ in order to illustrate the dependence of the pion loop contribution to $\left\langle r_{\pi}^{2}\right\rangle$ on $M_{c}$ and $\Lambda_{2}$, physical ranges for which are $0.2 \mathrm{GeV}<M_{c}<0.6 \mathrm{GeV}$ and $0.3 \mathrm{GeV}<\Lambda_{2}<0.7 \mathrm{GeV}$.

FIG. 5. In this figure we plot $\left[\hat{r}_{\pi}^{2} /\left(r_{\pi}^{2}\right)^{\text {GIA }}-1\right]$ as a function on $m_{\pi}$, in $\mathrm{MeV}$, in order to illustrate the onset of the pion-loop induced $\ln m_{\pi}$ divergence of $\left\langle\hat{r}_{\pi}^{2}\right\rangle$. It is clear from this figure that even for $m_{\pi}=50 \mathrm{MeV}$ the pion-loop contributes $<25 \%$ of the charge radius. The dashed line is the fit of Eq. (75). 


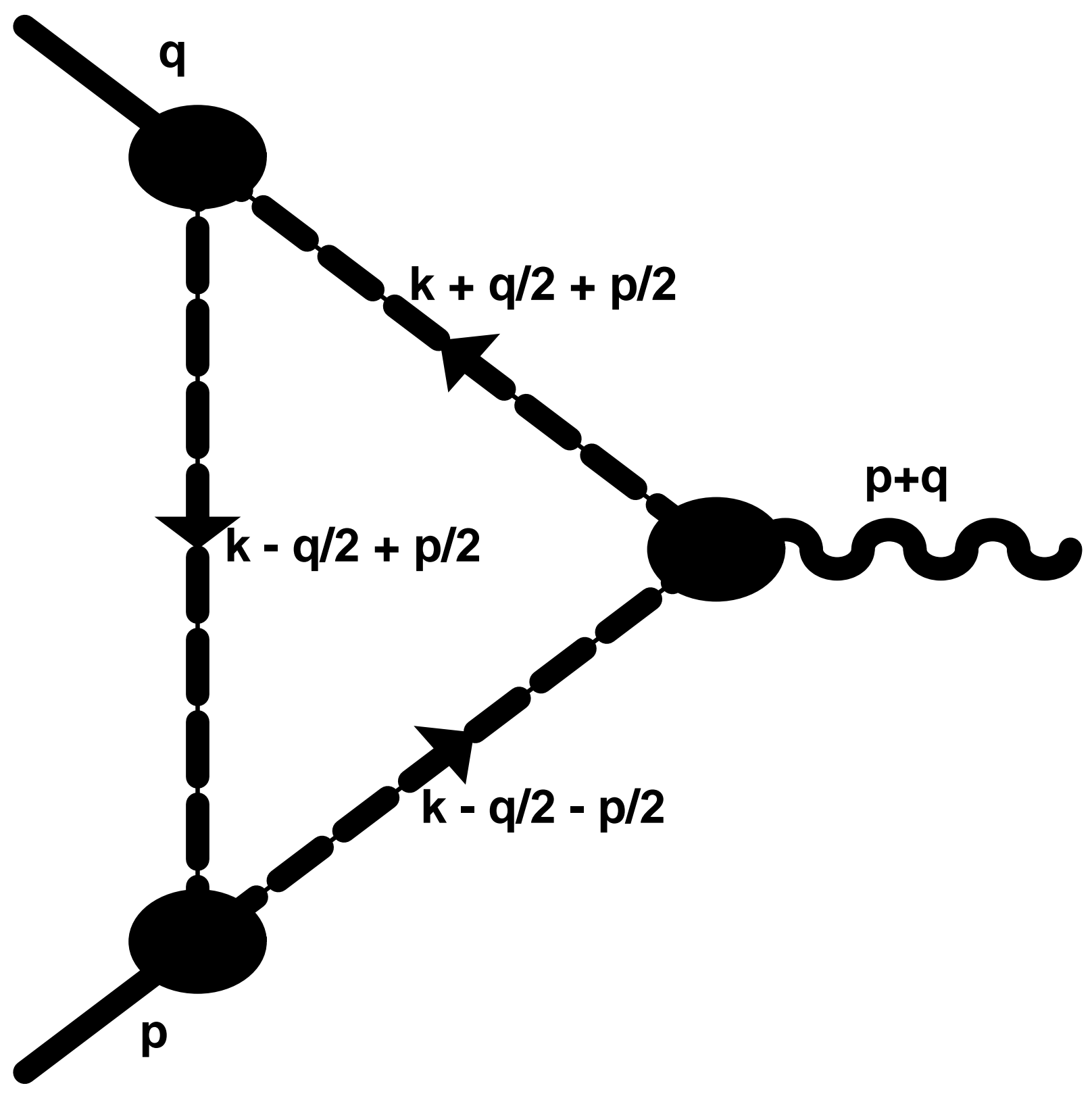


This figure "fig1-1.png" is available in "png" format from: http://arxiv.org/ps/hep-ph/9312243v1 
This figure "fig2-1.png" is available in "png" format from: http://arxiv.org/ps/hep-ph/9312243v1 


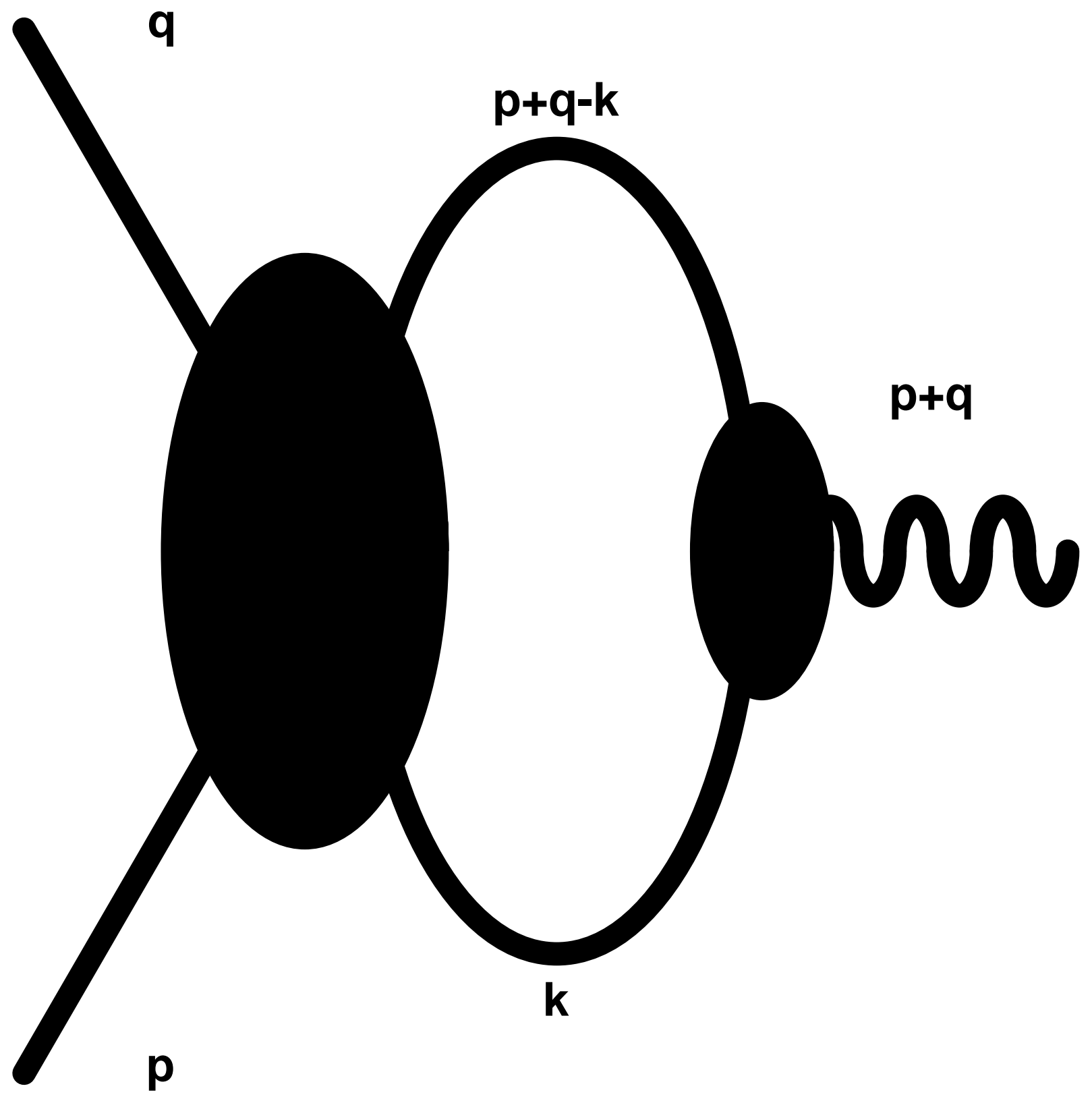


This figure "fig1-2.png" is available in "png" format from: http://arxiv.org/ps/hep-ph/9312243v1 
This figure "fig2-2.png" is available in "png" format from: http://arxiv.org/ps/hep-ph/9312243v1 


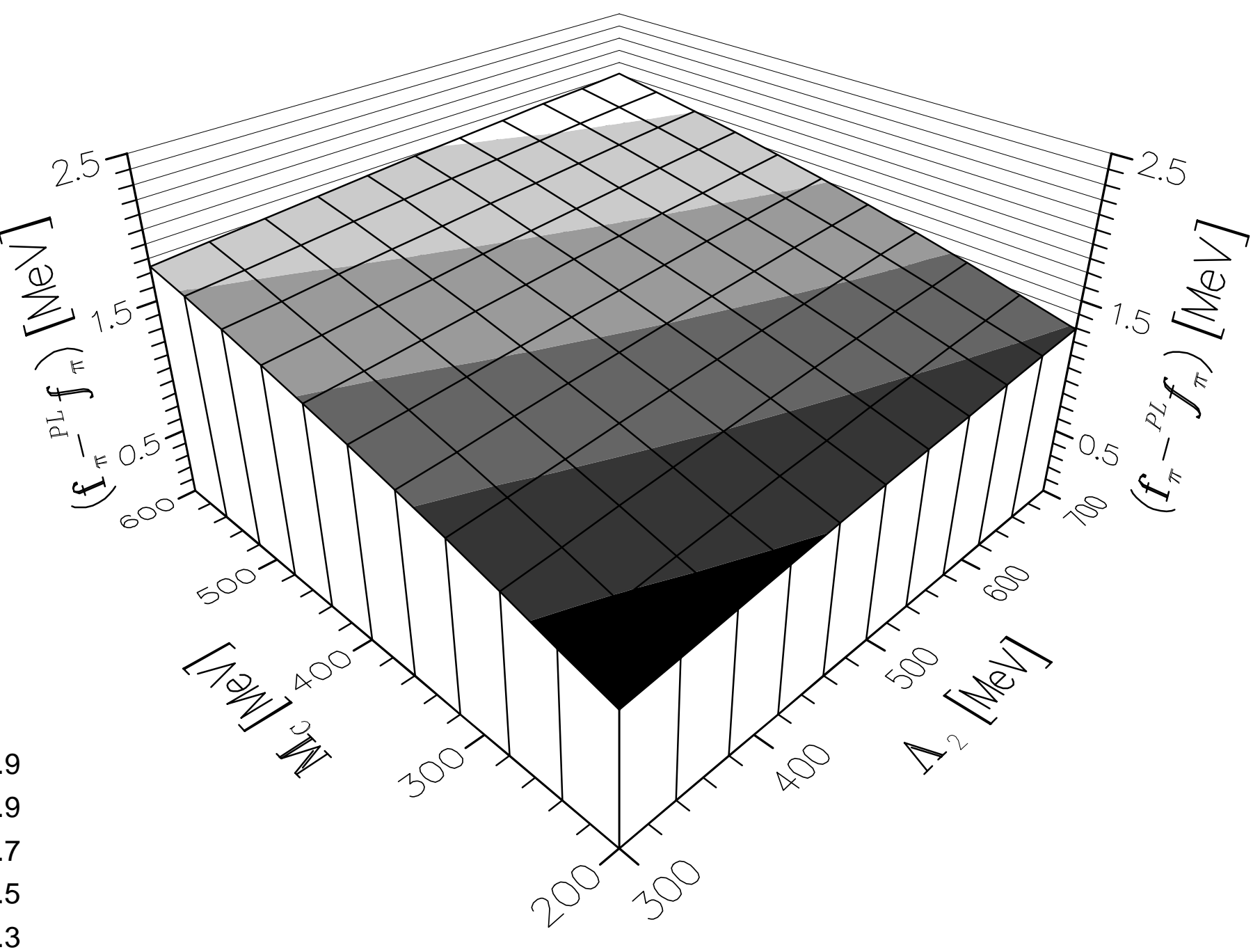


This figure "fig2-3.png" is available in "png" format from: http://arxiv.org/ps/hep-ph/9312243v1 
0.14

$0.12-\quad 0.14$

$0.10-\quad 0.12$

$0.08-\quad 0.10$

$0.06-\quad 0.08$

BELOW $\quad 0.06$

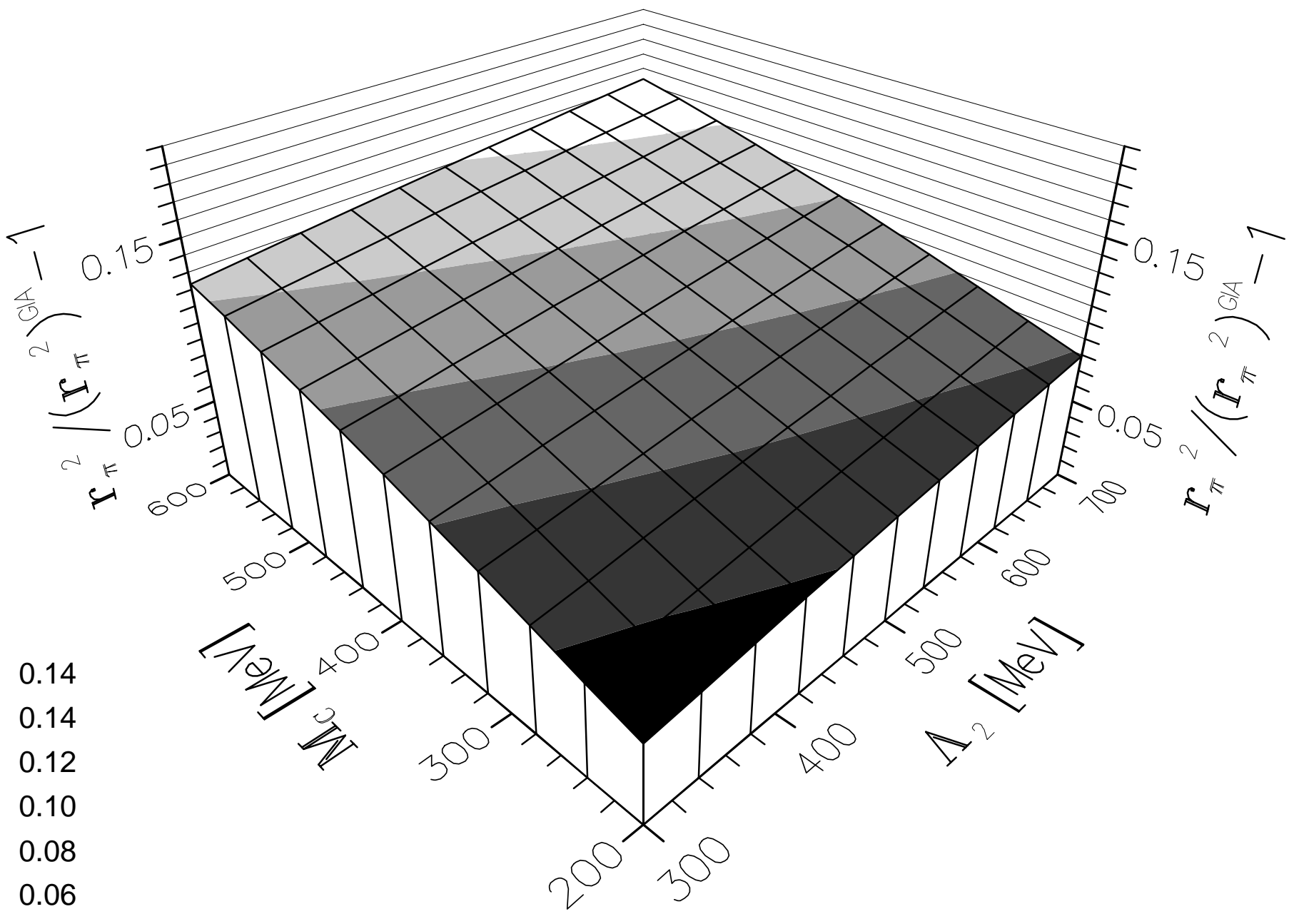




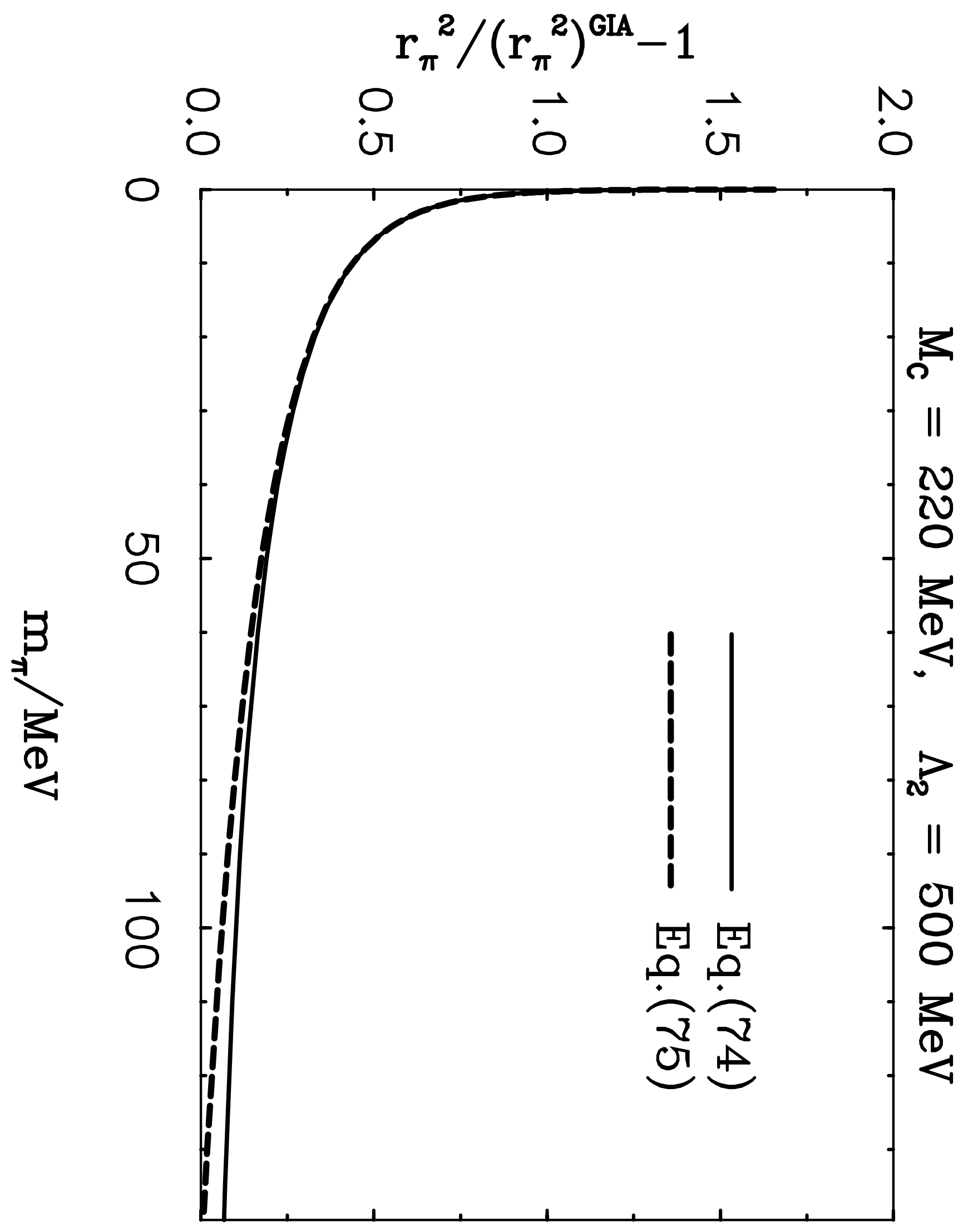

